\title{
On the morphology of peculiar ring galaxies
}

\author{
M. Faúndez-Abans and M. de Oliveira-Abans \\ Laboratório Nacional de Astrofísica, Caixa Postal 21, CEP:37.500-000, Itajubá, MG, Brazil
}

Received July 5; accepted October 13, 1997

\begin{abstract}
It is proposed that peculiar ring galaxies can be divided into five principal types according to the morphology of the ring and bulge, based on the visual inspection of 489 selected objects. Those objects have been named "peculiar" following the "Catalogue of Southern Peculiar Galaxies and Associations" by Arp \& Madore $(1986)^{1}$.
\end{abstract}

Key words: galaxies: peculiar - fundamental parameters - structure

\section{Introduction}

The number of studies of ring phenomenon in galaxies has grown in the last twenty years. The ring-like structures of $\mathrm{SB}(\mathrm{r})$ and $\mathrm{Sa}(\mathrm{r})$ galaxies with resonance rings, which are said to be related to orbital resonances in a bar or oval in the galactic gravitational potential (features also found in SAB galaxies) are also seen in wide-field, deep-sky images. The visual appearance of these objects will be hereafter referred to as "normal" ringed galaxies - NRG herein (for more on these objects, see Buta 1995, and references quoted therein). Other examples of NRG phenomena are: (a) early-type disk galaxies from $\mathrm{SO}^{+}$to $\mathrm{Sa}$, which show interesting structures such as nuclear, inner, and outer rings, (b) the normal ring category of spirals having stages $\mathrm{Sab}$ or later, and (c) Sa, SO/a and $\mathrm{SO}$ galaxies where the ring pattern is less prominent.

Extensive studies of ring sizes of NRG have been developed by de Vaucouleurs \& Buta (1980a,b), Buta \& de Vaucouleurs $(1982,1983)$, among others. Studying the metric properties of the inner ring structures of $\mathrm{S}(\mathrm{r})$ galaxies, Pedreros \& Madore (1980) found that ring diameters are rather highly accurate distance indicators. On the other hand, spiral galaxies which have had their arms tightly wound into nearly circular design, thus resembling ring-shaped structures (Arp \& Madore 1986 - hereafter

\footnotetext{
Send offprint requests to: M. Faúndez-Abans

1 Table 2 with its notes is only available electronically via anonymous ftp 130.79.128.5 or http://cdsweb.u-strasbg.fr
}

AM; Faúndez-Abans et al. 1992), are not considered as ring galaxies in this work.

In the last two decades, surveys, classification systems and statistics of ring galaxies have been made. As an example, in the seventies, Theys \& Spiegel (1976) classified northern ring galaxies in three groups: (1) RE-objects, which have crisp, elliptical rings with photographically empty interiors, with archetypes such as Arp 146, Arp 147 and VII ZW 466; (2) RN-objects, which have an elliptical ring with an interior off-center nucleus, archetype II HZ 4; and (3) RK-objects, characterized by rings with prominent condensations or knots, archetypes being I Zw 45 and Zw 28. In the eighties, Few \& Madore (1986) proposed a simple division of southern ring galaxies into two broad sub-types: P-type rings, which have a crispy knotty structure and often an off-center nucleus, and that are probably collisional (they happen to have a significant number of companions lying within a distance of about two ring diameters); and O-type rings, which have a smooth structure and a centrally located nucleus, no obvious companions, and that are likely to be resonant $(\mathrm{R}) \mathrm{S}$ galaxies.

The diffuse stellar component (nucleus) of ring-shaped galaxies as a classification parameter was introduced by Faúndez-Abans et al. (1992 - hereafter FAHR). On the other hand, the FAHR criterion also includes SB and Sa ringed galaxies (Hodge 1966), which are NRG. The category 6 of the "Catalogue of Southern Peculiar Galaxies and Associations" (Arp \& Madore 1977 and AM) also comprises a wide variety of ring morphologies, more than expected by the presently known classifications. The AM catalogue contains a variety of objects which display warped structures, off-center nuclei, face-on elliptical rings, disrupted rings and so on, features that could be a result of interactions such as (i) collisions ("galaxies run through each other"; see Appleton \& Marston 1997 for a recent work on optical, infrared, and radio continuum properties of a sample of collisional ring galaxies), (ii) tidal interactions ("one passes close to the other"), or (iii) merging (combination into one galaxy). To distinguish these objects from the NRG, we hereafter call these objects "Peculiar Ring Galaxies" (PRG, referred to as "peculiar" following AM catalogue's designation). It is also 
interesting to refer to the work of Naim \& Lahav (1997) on "what a peculiar galaxy is". We have purposedly included polar and saturn-like structures for completeness of the O-type rings of Few \& Madore (1986), even though O-type objects are perhaps resonant.

The main objective of this paper is to introduce a complementary view of PRG, based on the morphologies of the ring and the nucleus, and when possible, the scenario of the apparently close companions. The word "nucleus" was introduced by Theys \& Spiegel (1976) and Few \& Madore (1986); we will be using "bulge" herein, instead.

\section{Ring morphologies}

\subsection{Procedure}

The data base contains some ring-shaped objects of the AM catalogue, several ring-shaped galaxies of Few \& Madore (1986), FAHR, and Faúndez-Abans et al. (1994), and of the atlas of polar-ring galaxies of Whitmore et al. (1990).

The selected galaxies have been inspected on the $\mathrm{J}$ and R SRC/ESO Survey film copies to confirm their morphological types and, when possible, to make measurements of the bulge and ring diameters with a micrometer microscope. After careful inspection of the ring and bulge structures, they have been classified by their visual appearance (see Appendix A with Notes on the objects, available in electronic form only, at the Centre de Données astronomiques de Strasbourg - CDS). No photometric calibrations of the plate copies have been made; therefore, the measurements of the bulge's and ring's minor and major axes have not been made at the same isophotal level, but judiciously by eye, instead. This procedure often yields low-error dimensions for the ring, except in those cases when tidal distortions are large; on the other hand, the bulge's ellipticity is subject to the plate depth and influence of the disk and projected angular distance to the ring itself.

In a first step, when all morphological details had been taken into account, the galaxies were grouped in 29 different types of objects. Those which are NRG were then excluded from this classification. Next, the several morphological categories have been compressed into five, following the general behavior of the ring. Table 1 displays these five peculiar ring families.

\subsection{Types of rings}

The candidate objects display a wide variety of ring and bulge morphologies. Some structures could be remnants of interaction: rings with off-center bulge, external and internal rings with twisted principal axes, and warped rings. Others are in fact interacting ring galaxies showing bridges, filaments, crisps, plumes, and warped rings. It is not unusual to find knotty, smooth, polar, and elliptical rings.
Table 1. PRG morphologies

\begin{tabular}{l|c|l}
\hline Families of Rings & Code & Basic Structures \\
\hline Polar & P & $\begin{array}{l}\text { (a) Spindle } \\
\text { (b) Saturn } \\
\text { (c) Worm-like }\end{array}$ \\
Hoag & HL & $\begin{array}{l}\text { (a) Hoag } \\
\text { (b) Hoag-like }\end{array}$ \\
$\begin{array}{l}\text { Elliptical } \\
\text { (the warped behavior } \\
\text { is often present) }\end{array}$ & E & $\begin{array}{l}\text { (a) Knotted } \\
\text { (b) Smooth } \\
\text { (c) "Solitaire" }\end{array}$ \\
Irregular & I & \\
Centrally Smooth & CS & \\
\hline
\end{tabular}

\section{1) Polar rings}

It is generally accepted that the observed structure of polar ring galaxies come from accretion of matter from a nearby galaxy or by merging of a gas-rich companion. A study of the polar NGC 4650A by Sackett \& Sparke (1990) suggests that the central condensation is much smaller and less massive than the ring. They propose that a gas-rich dwarf galaxy may have disintegrated to form the ring instead of the gravitational stripping by/of a neighboring galaxy, as had been proposed earlier. This kind of object has been very well discussed by Whitmore et al. (1990), who present a photographic atlas and describe its appearance from a variety of viewing angles. They also report photometric and spectroscopic observations of several galaxies. A short and beautiful review can be found in Lucas (1993). For recent works on polar ring galaxies, the reader is referred to Reshetnikov (1997), Reshetnikov et al. (1995, 1996, and 1997), Hagen-Thorn \& Reshetnikov (1997) and references quoted therein.

There are three basic structures that can be associated to this family:

(a) Spindle. The traditional polar ring galaxies. These objects appear to have spindle-shaped bulges (the S0 galaxy) with a ring nearly perpendicular to the galactic major axis (polar ring).They can be further subdivided into two basic structures: (i) Objects with a well-defined ring without or with very faint external material, archetypes being ESO 415-G26 and HRG 19502, and (ii) objects which show rings with prominent outer extended material; archetypes are A0136-0801, AM 0226-320. The reader may also refer to the 
catalogue of box- and spindle-shaped objects by Jarvis (1986).

(b) Saturn. These objects have a spherical bulge with a bright round ring. It is proposed to also classify as saturn-type those galaxies of which bulge is a little elliptical. It is very likely that the saturn-like objects are only an effect of viewing angle, as displayed in Fig. 1 of Whitmore et al. (1990) for polar ring galaxies. Archetype is HRG 54103.

(c) Worm-like. A long worm-like bulge is the main characteristics. Whitmore et al. (1990) suggest that they are possible candidates for polar-ring galaxies. The ring around the bulge generally shows one knot with bulge-like dimensions, just as the "solitaire" ring galaxies (see below). Archetypes are ESO 199-IG12 and HRG 32501.

\section{2) Hoag rings}

These objects are characterized by a circular ring around the bulge. There are two kinds of objects in this family:

(a) Hoag. The name comes from Hoag (1950), who discovered an object which he described as a "perfect halo" surrounding a small diffuse bulge. At a first glance, these objects appear to be planetary nebulae because of the circular symmetry and the dimensions of the bulge (see also Brosch 1985, 1987).

(b) Hoag-like. These objects have Hoag-like morphology with the following differences: (i) some of them have low ellipticity bulges (Archetype FM 59-12), (ii) others, a comparatively large spherical bulge, archetypes FM 327-23 and FM 235-17. It is possible that some spindle-likes and saturns, when seen almost end-on, would appear as Hoag or Hoag-like.

\section{3) Elliptical rings}

Some of these objects are classified as RN by Theys \& Spiegel (1976), and as P objects by FM. In general, they show an off-center bulge, but in a few cases it is possible to discern a centered bulge with an elliptical ring, possibly inclined $30^{\circ}$ to $60^{\circ}$ to the line-of-sight. The ellipticals is a large family, where warped rings are often present. Three basic structures can be discerned:

(a) Knotted. The objects in this category have knotted rings and may be evidence of ring deformation as a result of tidal interaction; they may be undergoing star formation. In some cases, an internal elliptical ring is also present. Examples of these objects are HRG 35002, HRG 03401, and AM 2134-471.

(b) Smooth. They have either centered or off-centered bulge. Clear evidence of collision can be seen in some of the latter. Examples of these objects are:
HRG 13801, which also shows a bridge of material extending within the intruder, and HRG 08901, which is also interacting but displays no visual evidence of a bridge.

(c) "Solitaire". The bulge is on the ring, resembling a one-diamond finger ring called "solitaire" ("singleknotted ring"). The ring generally looks smooth and thin on the opposite side of the bulge, but there are a few cases where the ring appears broken on this opposite side. A few galaxies show a filamentary ring structure. The intruders may be elliptical to various degrees. Archetypes are FM 251-47 and FM 188-15.

\section{4) Irregular}

Several galaxies show irregular distortions in their structures which resemble pseudo-rings, arms, and curved tails.

\section{5) Centrally smooth rings}

These objects are knotted ring-like structures without an apparent bulge. The term has been originally introduced by Appleton \& Struck-Marcell (1996) and have been adopted in this work. Recently, Appleton et al. (1996, see also references quoted therein) have studied the northern archetype VII Zw 466 and found evidence of tail interaction, where one companion galaxy points to the intruder, which must have collided with VII Zw 466. A southern archetype is HRG 50201.

\section{Results and discussion}

The visual classification is important to group the galaxies in families, so that one may study the different formation processes (types of interactions) to understand their dynamics and structural evolution. In other words, each family should be triggered by one or more physical phenomena such as close encounters, collisions, or mergers. There are some exceptions to those scenarios, such as some very symmetric features in ring galaxies that may not be explained by merging. An example is the case of HRG 54103 , for which an alternative explanation may be that material rotating with different periods would settle in the ring (Barnes 1996). What about the different kinds of progenitors and interaction scenarios of PRG formation? Numerical models can explain some morphologies either by close encounters (gravitational perturbation) or collision (physical contact of some components of the interacting galaxies) - see e.g. Toomre (1969, 1977, 1978, 1985); Toomre \& Toomre (1973); Lynds \& Toomre (1976).

In summary, one may divide the ring galaxies into two main classes, namely "NRG" (ringed objects due to 
resonant trapping of matter) and "PRG" (often collisional).

The projected ring families displayed in Table 1 are sufficiently broad to include all known PRG. We have purposely included O-type galaxies because they are morphologically interesting, in contrast to the other NRG. Over 20000 galaxies have information on classification in the RC3 (de Vaucouleurs et al. 1991), but a few show ringshaped structures which have not been cited in the catalogue. In addition, there are galaxies which have not been classified because many have unusual morphologies due to interaction. How many dramatically distorted interacting systems show ring or pseudo-ring structures? There have been no systematical studies in the literature yet. In our sample, a few objects of this class with definite rings have been included in the Irregular family.

The warped rings are objects undergoing strong interaction, they may exhibit perturbed pseudo-rings, some probably being interaction-remnants. The smooth elliptical rings show both centered and off-centered bulges; small evidence of probable star forming regions is present in some objects, in contrast to the richness of HII regions displayed by the knotted elliptical rings. The solitaire objects show either smooth or smooth plus crispy rings, and no relation with the intruder's morphology has been found, which can be elongated, spherical or irregular.

Table 2 displays basic data on the 489 studied galaxies (available in electronic form only, at CDS). The different columns show: (1) the name of the object ("OA" refer to objects found during inspection of the DSS's disks); (2) other designation; (3) and (4) galaxy coordinates for the 2000 equinox (the quoted coordinates are expected to be good to 1 arcsec each; only HRG 00502 has a larger error); (5) the ring family classification; $\mathrm{N}, \mathrm{s}$ and $\mathrm{r}$ ? stand for NRG, spiral and probable ring existence, respectively, a blank space meaning the object has not been classified; (6) to (9) for illustrative purposes, the bulge's and ring's major and minor axes in arcsec, respectively; and (10) note running number (see Appendix A, also at CDS). Excluding the N, s and not-classified galaxies, the ellipticals comprise $46 \%$ of the quoted PRGs, followed by the irregulars and polars, with $14 \%$ and $12 \%$, respectively (see Table 3). The axe's measurements have been performed with a micrometer microscope at the Pontificia Universidad Católica de Chile, with a precision of $0.25 \mathrm{~mm}$.

In this first stage, this work has been concerned only with the projected morphology. Structural studies based on detailed surface brightness measurements on direct CCD images of a selected sample of PRG are in progress; these will help in disentangling the different structures of PRG, allowing models including orientation to be tested. A spectral classification scheme of Polar rings and spectroscopic studies of other ring families are under development with other collaborators; these results will be published in a series of forthcoming papers.
Table 3. Percentual frequency of occurrence of each type

\begin{tabular}{lrr}
\hline Family & Number of objects & $\%$ \\
\hline P & 51 & 12.0 \\
P? & 26 & 6.1 \\
HL & 8 & 1.9 \\
HL? & 3 & 0.7 \\
E & 197 & 46.4 \\
E? & 6 & 1.41 \\
I & 59 & 13.9 \\
I? & 3 & 0.7 \\
CS & 8 & 1.9 \\
CS? & 1 & 0.2 \\
r? & 63 & 14.8 \\
& & \\
\hline
\end{tabular}

Acknowledgements. This work was partially supported by the Conselho Nacional de Desenvolvimento Científico e Tecnológico (CNPq, Brazil). The Departamento de Astronomía y Astrofísica of the Pontificia Universidad Católica de Chile has kindly made available their laboratories and computational facilities. This research has made use of the NASA/IPAC Extragalactic Database (NED) which is operated by the Jet Propulsion Laboratory, Caltech, under contract with the National Aeronautics and Space Administration. We would like to thank the anonymous referee for his helpful comments and suggestions.

\section{References}

Appleton P.N., Charmandaris V., Struck C., 1996, ApJ 468, 532

Appleton P.N., Struck-Marcell C., 1996, Fund. Cos. Phys. 16, 111

Appleton P.N., Marston A.P., 1997, AJ 113, 201

Arp H.C., Madore B.F., 1977, Q. Jl. R. Astron. Soc. 18, 234

Arp H.C., Madore B.F., 1986, Catalogue of Southern Peculiar Galaxies and Associations, Vol. I, Positions and Descriptions, Clarke-Irwin, Toronto

Barnes J., 1996 (private communication)

Brosch N., 1985, A\&A 153, 199

Brosch N., 1987, Mercury, Vol. XVI, Number 6, 174

Buta R., 1995, ApJS 96, 39

Buta R., de Vaucouleurs G., 1982, ApJS 48, 219

Buta R., de Vaucouleurs G., 1983, ApJS 51, 149

de Vaucouleurs G., Buta R., 1980a, ApJ 85, 637

de Vaucouleurs G., Buta R., 1980b, ApJS 44, 451

de Vaucouleurs G., de Vaucouleurs A., Corwin H.G., et al., 1991, Third Reference Catalogue of Bright Galaxies. New York, Springer-Verlag

Faúndez-Abans M., Hertling G.P., Ramírez A.C., 1992, A\&AS 94,245

Faúndez-Abans M., Cuevas H.L., Hertling G.P., 1994, A\&AS 104,1

Few J.M.A., Madore B.F., 1986, MNRAS 222, 673

Hagen-Thorn V.A., Reshetnikov V.P., 1997, A\&A 319, 430

Hoag A.A., 1950, ApJ 55, 170 
Hodge P.W., 1966, The Physics and Astronomy of Galaxies and Cosmology. McGraw-Hill Book Co., New York

Jarvis B.J., 1986, AJ 92, 65

Lucas R.A., 1993 Mercury, Sept.-Oct., p. 6

Lynds R., Toomre A., 1976, ApJ 209, 382

Naim A., Lahav O., 1997, MNRAS 286, 969

Pedreros M., Madore B.F., 1981, ApJS 45, 541

Reshetnikov V.P., 1997, A\&A 321, 749

Reshetnikov V.P., Hagen-Thorn V.A., Yakduleva V.A., 1995, A\&A 303, 398

Reshetnikov V.P., Hagen-Thorn V.A., Yakduleva V.A., 1996, A\&A 314, 729

Reshetnikov V.P., Sotnikova N., 1997, A\&A 325, 933

Sackett P.D., Sparke L.S., 1990, ApJ 361, 408
Theys J.C., Spiegel E.A., 1976, ApJ 208, 650

Toomre A., 1969, ApJ 158, 899

Toomre J., 1972, Bull. AAS 4, 213

Toomre A., Toomre J., 1973, Scientific American, Dec., p. 39

Toomre A., 1977, in Evolution of Galaxies and Stellar Population, Larson R.B. and Tinsley B.M. (eds.). New Haven: Yale University Observatory, p. 401

Toomre A., 1978, IAU Symp. No. 79, Longair M.S. and Einasto J. (eds.). Reidel, Dordrecht, Holland, p. 109

Toomre A., 1985, Lectures on Galaxy Dynamics, III Advanced School of Astrophysics, IAGUSP, Brasil

Whitmore B.C., Lucas R.A., McElroy D.B., et al., 1990, AJ 100, 1489 